\title{
PROBLEMA, OBJETIVOS Y ANÁLISIS DE DATOS: UNA REFLEXIÓN METODOLÓGICA EN LA INVESTIGACIÓN PSICOLÓGICA
}

\author{
Problem, objectives and data analysis: \\ A methodological reflection in psychological research
}

\author{
Carlos Ramos-Galarza* \\ Pontificia Universidad Católica del Ecuador
}

\begin{abstract}
Resumen
En este artículo se analiza la relación existente entre los elementos que componen la estructura de una investigación: la pregunta, hipótesis, objetivos y análisis estadístico. Se hace énfasis en la interrelación que deben poseer cada uno de estos elementos, los cuales se convierten en fundamento de cada fase que se va construyendo en una investigación. Al hablar de la pregunta de investigación se hace mención al cuestionamiento científico que surge luego de haber identificado un vacío en una línea de investigación. El siguiente elemento es la hipótesis y en el artículo se profundiza en las hipótesis de tipo descriptivo, correlación, comparación de grupos, causales y de intervención. Al hablar de los objetivos de investigación se hace mención al grado de complejidad que posee cada verbo que se utiliza y su relación con el análisis estadístico que se ejecutará posteriormente. Se cierra el trabajo reflexionando sobre la necesidad de que una investigación sea configurada como una maquinaria, en donde cada engranaje debe permitir que el estudio ejecutado tenga una presentación armoniosa.
\end{abstract}

Palabras clave: Análisis estadístico, hipótesis, investigación en psicología, método hipotético deductivo, objetivos de investigación, pregunta de investigación.

\begin{abstract}
In this article, the existing relationship between the elements that compose the structure of a research: question, hypotheses, objectives and statistical analyses are analyzed. Emphasis is placed on the interrelation that each of these elements must possess, which become the foundation of each phase that is being built in an investigation. When talking about the research question, mention is made of the scientific question that arises after having identified a gap in a line of research. The next element into consideration are the hypotheses, this article deep into the hypotheses of descriptive type, correlation, comparison of groups, causality and intervention. When comes to talk about the research objectives, there will be mentioned the degree of complexity of each verb that must be used according to the statistical analyses that will be conducted. The work is closed considering the need for an investigation which must be configured as a machinery, where each gear must allow the study executed have a harmonious presentation.
\end{abstract}

Keywords: Statistical analysis, hypothesis, research in psychology, hypothetical deductive method, research objectives, research question.

\footnotetext{
* Profesor Principal de la Facultad de Psicología, Pontificia Universidad Católica del Ecuador. caramos@puce.edu.ec

https://orcid.org/0000-0001-5614-1994
} 


\section{INTRODUCCIÓN}

En mi rol de profesor de investigación, guía de tesis y revisor de artículos científicos en el contexto de la ciencia psicológica, me es frecuente encontrar propuestas de investigación en las que se pasa por alto la estrecha relación que deben poseer los elementos esenciales que componen un proyecto de investigación: la pregunta de investigación, hipótesis, objetivos y análisis estadístico. En tal sentido, en este trabajo analizaré la relación que existe entre estos elementos de una investigación, con la finalidad de aportar en la comprensión de la estructura que debe poseer una propuesta científica en el contexto psicológico.

Las razones que motivan el tratar este asunto, tienen que ver con la revisión que realizo frecuentemente de propuestas de investigación en psicología, tesis de grado y post grado y como revisor de artículos, en donde he encontrado que no siempre los autores del estudio, tienen presente la lógica existente de la relación entre la pregunta de investigación, hipótesis, objetivos y análisis estadísticos, y se los plantea como elementos aislados y en muchas de las veces, he encontrado que ni siquiera existe correspondencia y se observa que la pregunta de investigación hace una determinada propuesta, las hipótesis otro punto y, ni se diga de los análisis estadísticos, que proponen otra cosa, casi como si pertenecieran a proyectos de investigación distintos. Por tanto, en este artículo quiero proponer un primer acercamiento a lo que sería una representación lógica de la relación entre estos elementos, lo cual servirá como un documento de uso pedagógico en la formación de investigación en psicología, así como un aporte para quienes nos interesamos en el día a día, resolver problemas mediante el método científico.

\section{Pregunta de Investigación}

Es común que un estudiante que esté realizando una investigación, cuando comenta la idea que tiene sobre su proyecto, lo primero que escucha de su profesor guía es "cuéntame cuál es tu pregunta de investigación", momento en el cual, todo su interés de investigación se resume al cuestionamiento que está por expresar, en donde debe definir si se trata de una investigación con alcance descriptivo o explicativo, de igual manera, si se podrá resolver con una investigación con una metodología cuantitativa o cualitativa, razón por la cual, la pregunta de investigación es una parte esencial de la investigación, que delimitará el resto de fases que se transitarán metodológicamente.

Este elemento constituye la médula del proyecto de investigación y es resultado de la revisión de la construcción teórica y estudios previos que se han realizado en una determinada línea de investigación. Además, surge luego de que el investigador ha realizado un análisis sensitivo del estado del conocimiento y de la realidad en la que se encuentra. Entonces, luego de que se ha cumplido con dicho proceso, emerge una pregunta que, mediante su respuesta con un método de investigación, logrará resolver algún aspecto teórico de la línea de investigación de interés o una necesidad de la realidad circundante al investigador.

Una pregunta de investigación debe ser planteada con tal transparencia que tan solo con leerla, invite a visualizar su metodología válida y viable (Garrocho \& Pozos, 2012, p. 2), en donde, en el caso del método cuantitativo, interés central de este trabajo, permita realizar mediciones para comprobar supuestos de interés en la ciencia psicológica. Además, existen diversas fuentes de las cuales puede emerger una pregunta de investigación interesante, que pueden ser la misma experiencia del investigador, lectura de fuentes especializadas y otras. En la tabla 1 se presentan varias fuentes de las que pueden emerger las preguntas de investigación. 
Tabla 1.

Fuentes para el surgimiento de una pregunta de investigación

\section{Fuentes de una pregunta de investigación}

Experiencia

Bibliografía especializada

Aparición de nuevas tecnologías

Escepticismo y observación

Fuentes externas

\section{Descripción}

Se basa en la experiencia que posee el investigador de acontecimientos previos en contexto profesional.

La pregunta surge por la revisión crítica de libros o artículos científicos que permiten identificar los aspectos no resueltos todavía en una línea de investigación.

En este tipo de fuente lo avances tecnológicos implican el surgimiento de preguntas de investigación, por ejemplo, cuestionamientos que tengan que ver con la adaptación de los instrumentos de evaluación psicológica a dispositivos tecnológicos actuales.

Las preguntas de investigación de esta fuente tienen que ver con analizar de forma crítica las teorías existentes y ponerlas a prueba mediante una investigación. En estos cuestionamientos el investigador debe ser altamente sensitivo al observar los procesos de evaluación, diagnóstico e intervención psicológica, para que pueda identificar algún elemento contradictorio y surja una pregunta de investigación que lo resuelva.

Las reuniones con expertos, la navegación en internet, el surgimiento de nuevos fenómenos humanos, entre otros, son fuentes de donde puede emerger una interesante pregunta de investigación.

Adaptado de: Garrocho \& Pozos (2012)

Además de la descripción previa, el investigador debe procurar que la pregunta de investigación que proponga, cumpla con características como: actualidad, aporte al conocimiento, viabilidad, factibilidad, pertinencia, precisión, interés y ética; las cuales le permitirán proponer una pregunta digna de ser respondida mediante una investigación (Acevedo, 2002; Arguedas, 2009; Doval, 2006; Manterola y Otzen, 2013).

Cuando se plantea una pregunta de investigación en el método cuantitativo se debe tener presente que sea direccionada y específica, que invite a realizar una medición de la realidad mediante la aplicación de reactivos accesibles al investigador. Una vez que se ha planteado una o varias preguntas de investigación, la siguiente fase que debe ser solucionada por el investigador es una respuesta tentativa, mediante una proposición afirmativa, conocida como hipótesis de investigación (para una revisión más extensa del planteamiento de la pregunta de investigación ver Ramos-Galarza, 2016).

\section{Hipótesis}

La hipótesis debe contar con la característica esencial de invitar a la medición de variables objetivas de la realidad, ya que aspectos que busquen subjetivaciones en la investigación, son clásicos del método inductivo, que será tratado en un siguiente trabajo. En el método hipotético deductivo, las hipótesis se plantean de forma a priori a la ejecución del estudio de campo del método cuantitativo (RamosGalarza, 2015).

Según Creswell (2014), las hipótesis son predicciones que los investigadores realizan acerca de los resultados que esperan sobre las relaciones entre las variables de interés. Son estimaciones basadas en la lógica numérica, sobre la población en base a los datos recolectados en la muestra de investigación. Para comprobar o refutar una hipótesis es necesario aplicar métodos estadísticos, en donde el investigador realice inferencias para explicar el comportamiento de una determinada variable para toda la población. En la figura 1 se presentan diversos tipos de hipótesis. 


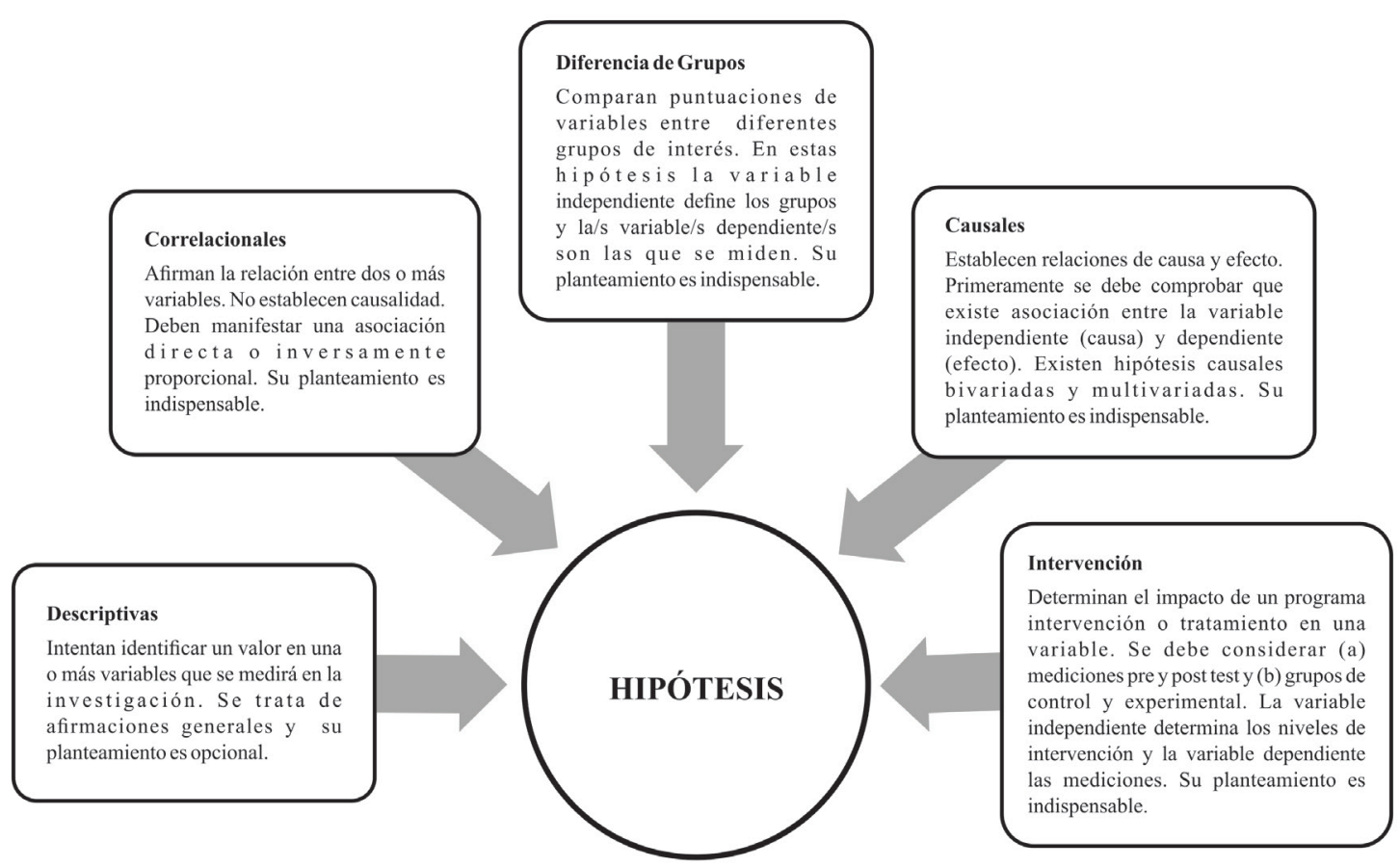

Figura 1. Tipos de hipótesis que se aplican en investigaciones en las investigaciones en Psicología.

Adaptado de Hernández, Fernández y Baptista (2014) y Ramos-Galarza (2017).

Además de los tipos de hipótesis descritos en la figura 1, clásicamente existe otro tipo, como lo es la hipótesis nula, que afirma la no existencia de diferencias entre grupos o no presencia de asociaciones entre las variables propuestas, por tanto, el fin de una investigación es rechazar la hipótesis nula, para comprobar alguna de las hipótesis presentadas previamente. Otro tipo de hipótesis son las hipótesis alternativas o hipótesis direccionales, en las cuales se plantean asociaciones diferentes a la hipótesis de investigación central (Creswell, 2014), por ejemplo, en estudios donde se verifican varios modelos de análisis factorial confirmatorio, se plantea una hipótesis alternativa para cada uno de ellos. Es importante mencionar que en una investigación se plantea el número de hipótesis que sean necesarias, no existe un número determinado, sino que, dependiendo de las preguntas de investigación se cuente, se plantearán hipótesis como una respuesta tentativa.

\section{Objetivos de Investigación}

Cuando se plantea un objetivo de investigación, se debe tener presente que cada verbo que se puede utilizar presenta un determinado nivel de complejidad, el cual se va incrementando, a la vez que engloba a otros de mayor simplicidad. Es como cuando en mi clase de investigación suelo realizar el ejercicio de analizar la canción de José José, que afirma que entre amar y querer existe una gran diferencia, no obstante, en muchas investigaciones que he revisado en mi quehacer psicológico, veo que se utilizan los verbos de manera indiscriminada, a pesar de que, amar y querer no es lo mismo. En tal sentido, el verbo explorar representa la menor complejidad para una investigación, que a su vez está englobado por otro de mayor alcance como lo es describir, hasta llegar al verbo determinar que podría implicar una mayor complejidad de la investigación, tal como se lo propone en la figura 2 . 


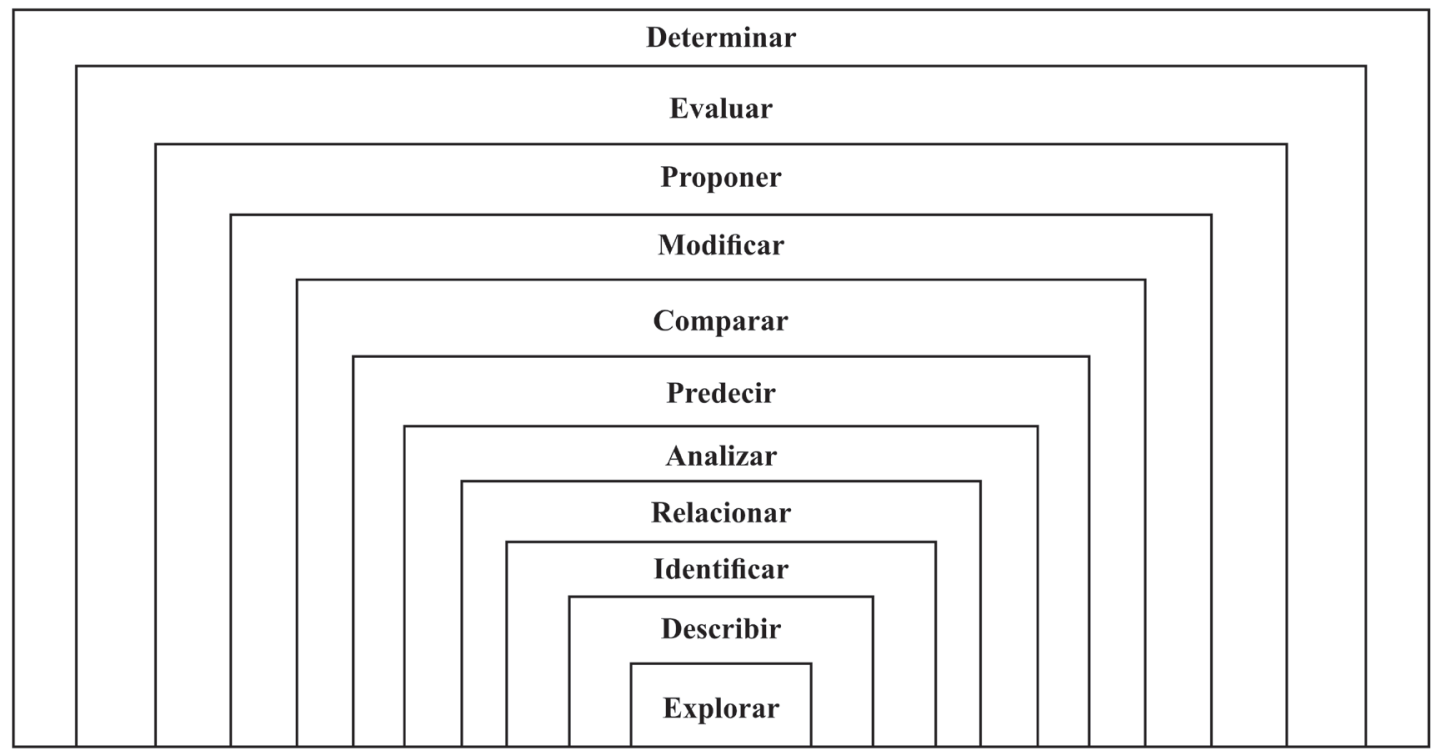

Figura 2. Complejidad de los verbos que se aplican en la investigación. Propuesta realizada en base a Ramos-Galarza (2014) y Hurtado (2008).

Según Hurtado (2008, p.37), plantear un objetivo es como "crear un manual de procedimientos administrativos para la supervisión de educación básica, que incorpore los aspectos legales, teóricos y didácticos señalados por el Ministerio de Educación”, es un error, ya que este tipo de trabajos no implican la ejecución de una investigación científica previa para lograrlos. Según el mismo autor, otro objetivo erróneo sería "desarrollar un libro de texto para el área de ciencias de la naturaleza, que incluya los contenidos y estrategias propuestos por el nuevo diseño curricular", ya que de igual manera, no invita a la ejecución de una investigación que implique un estudio de campo o algún tipo de componente empírico de datos.

En cuanto al uso de la taxonomía de Bloom como lineamiento para proponer objetivos de investigación, se debe tener presente que, en muchos de los verbos propuestos en dicha taxonomía, son más bien, actividades (por ejemplo, enumerar, escribir, memorizar, conocer, resumir, agrupar, opinar, etc.) más que verbos que impliquen la aplicación de una metodología de investigación. Una representación que podría aclarar esta afirmación es que un objetivo de aprendizaje podría ser el que un niño aprenda a escribir, no obstante, el escribir es una actividad operativa dentro de una investigación, mas no una investigación en sí (Hurtado, 2008).

Muchas de las veces los investigadores neófitos cometen un error en sus propuestas, ya que en ocasiones presentan una determinada pregunta de investigación, que no tiene concatenación con la hipótesis y menos aún con el objetivo y ni se diga con el análisis estadístico; conformando así, los clásicos artículos en los cuales, en la introducción se propone un tema, en la metodología algo totalmente nuevo, en los análisis estadísticos algo sin relación a la propuesta previa, en fin, proponiendo algo sin mucho sentido para el ojo científico.

Hurtado (2008, p. 46) propone como ejemplo de este típico error, cuando se plantea una pregunta como la siguiente "¿Cuál es la situación laboral del personal asistencial de los hospitales públicos de Santiago?" la cual debería tener un objetivo relacionado, sin embargo, un objetivo incorrecto sería "formular un programa dirigido a mejorar la situación laboral en el personal asistencial de los hospitales públicos de Santiago", lo cual estaría haciendo mención a diferentes investigaciones, dejando de lado la propuesta de armonía entre los elementos de una investigación presentada en este artículo. 
Si consideramos la figura 2, en la cual se presenta una propuesta de verbos para el planteamiento de objetivos, la pregunta previa, haría mención a una respuesta con un objetivo de tipo descriptivo, por ejemplo, utilizando los verbos explorar, describir e identificar, sin embargo, en el objetivo descrito previamente, se propone diseñar un programa, lo cual es una respuesta que busca modificar la situación que están viviendo los trabajadores, yendo así, más allá de la pregunta descriptiva exploratoria planteada inicialmente.

En cambio, de forma correcta se plantearía de la siguiente manera la pregunta: “¿Cuál es la situación del personal asistencial de los hospitales públicos de Santiago" y su objetivo con un alcance descriptivo sería: "describir la situación laboral del personal asistencial de los hospitales públicos de Santiago", de manera que, se mantendría la relación entre ambos elementos de la investigación (Hurtado, 2008, p. 47). En la tabla 2 se presenta un ejemplo en donde se puede identificar la relación entre los elementos planteados.

\section{El uso y abuso del verbo DETERMINAR}

El verbo determinar significa "llegar a saber una cosa a partir de los datos que se poseen, pronunciar una sentencia" (The Free Dictionary, 2018), "fijar los términos de una cosa, sacar conclusiones a partir de datos conocidos, sentenciar" (Word Reference, 2018), "despejar la incertidumbre sobre ello, establecer o fijar algo, indicar algo con exactitud" (Real Academia de la Lengua Española, 2018 a), "señalar la acción de establecer un tipo de dato, fijar o hacer claros los elementos de una situación, cosa o evento", "el determinar algo es hacerlo claro, poner los términos que lo delimitarán” (Definición ABC, 2018).

Como se puede observar en su definición, el verbo determinar sería el representante más puro de la investigación desde el paradigma positivista, ya que el determinismo clásico del método cuantitativo ortodoxo, busca determinar los diferentes factores causales, para controlarlos y manipularlos mediante el método experimental (Ramos-Galarza, 2015).

En tal sentido, es menester tener presente el significado de este verbo al momento de plantear una investigación, ya que muchas veces he sido testigo de propuestas de investigación con una metodología inductiva cualitativa, basada en narrativas de un grupo humano, que utilizan como objetivo general el verbo determinar para proponer el fin de la investigación, lo cual es una grave contradicción en la investigación. De igual manera, es un error plantear un objetivo que busque determinar un fenómeno y luego en el análisis estadístico proponer técnicas descriptivas de tendencia central o dispersión. En tal sentido, es indispensable que el investigador tenga una plena conciencia del verbo que utiliza al momento de plantear su objetivo, ya que, como se mencionó previamente, amar no es lo mismo que querer. 
Tabla 2.

Ejemplo de la relación entre los elementos de una investigación

\begin{tabular}{l} 
Pregunta de Investigación \\
\hline Pregunta con un nivel \\
descriptivo: \\
¿Cuáles son las características \\
de un fenómeno? \\
¿Cómo se manifiesta el \\
fenómeno? \\
¿Qué cantidad de personas \\
presentan el fenómeno? \\
Pregunta de comparación: \\
¿Cuáles son las diferencias \\
existentes [variable \\
dependiente o medida] entre \\
personas que presentan \\
[variable independiente, define \\
los grupos] el fenómeno (a), en \\
contraste de las personas que \\
presentan el fenómeno (b)?
\end{tabular}

Pregunta de correlación:

¿Cuál es la asociación existente entre la variable A y la variable $\mathrm{B}$ ?

Ojo, en este nivel de pregunta no se plantea variables independientes o dependientes.

Pregunta de predicción: ¿Cuáles es/son los (X ó variable independiente) factor/es etiológico/s de la condición (Y ó variable dependiente).

La teoría ... puede explicar la relación entre (variable independiente) y (variable dependiente), controlando los efectos de (variable de control)?

Pregunta de validación: ¿Qué propiedades psicométricas presentará una escala para valorar una determinada condición?

\section{Hipótesis Objetivos}

Su planteamiento es opcional, Se debe tener presente el uso es de tipo descriptivo o de verbos con un alcance exploratorio.

Opciones de hipótesis serían: fenómeno.

El fenómeno posee los Caracterizar los elementos que elementos (a), (b) y (c).

$\mathrm{Su} \quad$ planteamiento indispensable.

El grupo que presenta el fenómeno (b) tendrá mayor rendimiento en la condición [variable dependiente], a diferencia del grupo (a), que presentará menor rendimiento en la condición [variable dependiente].

$\mathrm{Su}$ planteamiento es Relacionar la variable.

indispensable.

La variable A ascenderá en su nivel cuando la variable B ascienda en su nivel.

La variable $\mathrm{B}$ descenderá en su nivel, cuando la variable A ascienda en su nivel.

$\mathrm{Su}$ planteamiento es indispensable.

El/los factor/es $X_{1}, X_{2}$ y $X_{3}$ explicarán la incertidumbre de la variable Y.

$\mathrm{Su}$ planteamiento

indispensable.

La escala presentará un adecuado valor de consistencia interna.

La escala ajustará de manera adecuada en $\mathrm{x}$ factores.

La escala correlacionará de forma significativa con otro instrumento que mida un constructo similar. descriptivo.

Identificar el porcentaje de personas que presentan el fenómeno.

\section{Análisis Estadístico}

Se deben aplicar técnicas estadísticas a nivel descriptivo:

Media, moda, mediana, porcentajes, frecuencias, desviación estándar, otras. configuran el fenómeno.

Comparar el rendimiento en la condición [variable dependiente] en personas con [variable independiente define los grupos] la condición (a) y condición (b).

Se deben aplicar técnicas estadísticas de tipo inferencial que permitan comparar grupos: $t$ de Student, U de Mann Whitney, ANOVA, KruskalWallis y otros.

Se deben aplicar técnicas estadísticas de correlación:

Correlación de Pearson o Spearman, chi cuadrado, Tau-b de Kendall y otros.

Predecir la incertidumbre de la variable $\mathrm{Y}$, al considerar como factores de causalidad $\mathrm{X}_{1}, \mathrm{X}_{2}$ $\mathrm{y} \mathrm{X}_{3}$.

Se deben aplicar técnicas estadísticas inferenciales de predicción:

regresión lineal, regresión múltiple, regresión logística binaria, regresión logística, multinomial y ordinal, Odds Ratio y otros.

Se deben aplicar técnicas estadísticas psicométricas: evaluación de la condición $\mathrm{X}$. Modificar la escala X para valorar una determinada condición.

Evaluar las propiedades psicométricas de la escala $\mathrm{X}$ para valorar una determinada condición.
Alfa de Cronbach, Kappa de Cohen, análisis factorial, correlación, otros.

Nota. En los análisis predictivos, la variable independiente es la causa del fenómeno (no depende de ninguna variable) y se la representa con X. La variable dependiente es el efecto que depende de la causa (de la variable independiente), se lo representa con Y. En los estudios experimentales la variable independiente define los niveles de manipulación y la variable dependiente es la variable que recibe la influencia de la manipulación intencionada y es la variable que se mide (Field, 2009). 
Al momento de plantear una investigación dentro del modelo positivista o post-positivista (en un siguiente artículo ya trabajaré la belleza metodológica hermenéutica del paradigma constructivista), el científico en psicología no solo debe dominar la pregunta de investigación, hipótesis y objetivos, sino que, es indispensable, desde mi entender, que se domine también el proceso de análisis estadístico, porque si se espera, como alguna vez lo expresó un colega en un encuentro de investigadores, "no es necesario que en psicología manejemos el análisis estadístico, porque podemos contratar expertos en estadística que hagan ese trabajo". Es por esta razón que el investigador psicológico debe contar con un importante dominio de todos los elementos que engloban a la investigación, sino, podría cometer errores ingenuos, en donde proponga objetivos o hipótesis que no tienen una correcta relación con los análisis estadísticos que puedan ser propuestos por un experto contratado.

\section{Análisis Estadístico}

Dentro de la hermosa epistemología del positivismo y post-positivismo, la estadística contiene el procedimiento que permite al científico la comprobación de hipótesis a través de los análisis numéricos ejecutados y, proponer así, mediante el método científico, el avance de una determinada ciencia, tal como lo hacemos día a día en la ciencia psicológica (Ramos-Galarza, 2017 a y b).

Uno de los aspectos más enriquecedores de la estadística en la psicología, es que "no analiza individuos aisladamente, sino conjuntos de ellos, conjuntos a los que define de acuerdo a ciertas características que elige deliberadamente", sino que, en base a comparaciones y relaciones entre diferentes factores, busca entender los comportamientos, procesos y fenómenos sociales a nivel de la población (Bologna, 2013, p. 19).

En el análisis estadístico existe una gran cantidad de procesos cuantitativos que enriquecen la investigación, por ejemplo, se cuenta con procesos estadísticos descriptivos e inferenciales que permiten al científico ejecutar una diversidad de investigaciones de interés para la psicología.
Como se presentó en la tabla 2 el análisis descriptivo incluye mediciones como la media, moda, mediana, porcentajes, frecuencias, desviación estándar y otras que permitan identificar medidas de tendencia central y dispersión. Estos análisis sirven para caracterizar los valores numéricos de las variables y se aplican cuando la pregunta de investigación, hipótesis y objetivos de investigación tienen un alcance descriptivo exploratorio.

En cuanto a los análisis estadísticos inferenciales, en la psicología se pueden aplicar procedimientos cuantitativos de tan alto nivel como los que se aplican en ciencias exactas como la economía (Ramos-Galarza, 2017 a). Al igual que en el análisis descriptivo, en el análisis inferencial existe una estrecha relación con las preguntas de investigación, hipótesis y objetivos, los cuales deben tener una connotación explicativa y permitir al científico realizar análisis como correlación entre variables, predicciones, comparaciones entre grupos, impacto de intervenciones, detección de factores de riesgo/protección o de variables mediadoras/ moderadoras, ajustes de modelos explicativos, análisis de propiedades de validez y confiabilidad, entre otros procedimientos que hacen a la psicología una ciencia con gran riqueza para la aplicación de las técnicas científicas cuantitativas. Es importante mencionar que actualmente existen varios paquetes estadísticos que permiten al investigador la automatización de los análisis, haciendo así, más accesible la aplicación de la estadística en la investigación psicológica (Castañeda, Cabrera, Navarro, \& Vries, 2010).

\section{Correlación Espuria}

Un concepto que es necesario tener presente en la práctica científica psicológica es la correlación espuria -falsa, fingida- (Real Academia de la Lengua Española, 2018 b) que en ocasiones es común ver en investigaciones, refiriéndome de manera específica a estudios en donde se ve que se ingresan en el análisis estadístico y en los resultados del manuscrito de reporte de la investigación, todas las variables que se midieron en el estudio de campo, en una especie de licuadora, a la espera de obtener algo interesante, claro en el mejor de los casos, porque en ocasiones sólo se ven cuadros de correlación que podrían sorprender a un ojo ingenuo que se sorprende por ver números inexplicables en un 
artículo, no obstante, están cayendo en el más garrafal de los errores.

Como ejemplo de este concepto, se presenta a continuación en la tabla 3 , una correlación que representa una relación estadísticamente significativa y carente totalmente de lógica o significación.

Tabla 3 .

Ejemplo de Correlación Espuria

\begin{tabular}{llr}
\hline & & Edad \\
\hline Género & Correlación & $-.325^{* *}$ \\
& Sig. (bilateral) & $<.001$ \\
& $\mathrm{~N}$ & 121 \\
\hline
\end{tabular}

**. La correlación es significativa en el nivel .01

Según la tabla 3 ,existiría una relación inversamente proporcional estadísticamente significativa, en donde, cuando la variable $\mathrm{X}$ asciende numéricamente, la variable $\mathrm{Y}$ desciende, o viceversa. Entonces, esta relación numérica podría significar que (ojo, son datos de una investigación real), cuando la variable edad asciende, la variable género desciende, lo cual sería totalmente descabellado, ya que el género de un ser humano no depende de su edad, sino del intercambio cromosómico al momento de la fecundación (Carlson, 2014).

Este error en el análisis estadístico es muy común verlo en investigaciones en donde los responsables de estos estudios no tienen una conciencia plena de la relación de los análisis de datos con el planteamiento de las preguntas o hipótesis de investigación. En el caso previo, la hipótesis que motive este análisis sería incomprensible, no obstante, lo absurdo de este ejemplo, proyecta la necesidad de que los investigadores en psicología tengamos presente que, cada análisis estadístico tiene que servir para comprobar o refutar la hipótesis planteadas y si existe algún análisis extra o que el investigador no sepa para que se encuentra en el reporte de la investigación, pues estará metiendo en la licuadora todo lo medido y tratará de rellenar su artículo de manera artificial.

\section{DISCUSIÓN: LA RELACIÓN ENTRE LA PREGUNTA, HIPÓTESIS, OBJETIVOS DE INVESTIGACIÓN Y ANÁLISIS ESTADÍSTICO DE UNA INVESTIGACIÓN}

En este artículo se ha intentado realizar una propuesta para esclarecer que en una investigación psicológica desde el método cuantitativo (como ya lo dije, en otro artículo trataré la belleza del paradigma constructivista y su metodología de investigación cualitativa), debe existir una coherencia entre los diferentes engranajes que corresponden la maquinaria de una investigación, es decir, cada elemento debe ser correspondiente con el subsiguiente. De esta manera, una vez que el investigador proponga la pregunta de investigación, debe realizar una propuesta de respuesta tentativa a esa indagación que será la hipótesis que guíe su investigación, lo cual dará como resultado el siguiente elemento de la maquinaria, el objetivo de la investigación y de manera lógica, permitirá el planteamiento del análisis estadístico correspondiente, tal como se lo presentó en la tabla 2.

Con el análisis presentado en este artículo, se deja en evidencia que cada elemento de la investigación debe estar interrelacionado con sus elementos previos y no de forma mágica o desde la mente de un iluminado, que conoce cuál es el análisis estadístico para comprobar las hipótesis planteadas o para responder las preguntas de investigación, sino que, existe una lógica que debería estar cimentando toda estructura de una investigación, tal como se lo presenta en la figura 3 , en donde se sintetiza todo lo mencionado en este trabajo y permite identificar el funcionamiento de la maquinaria de una investigación. 


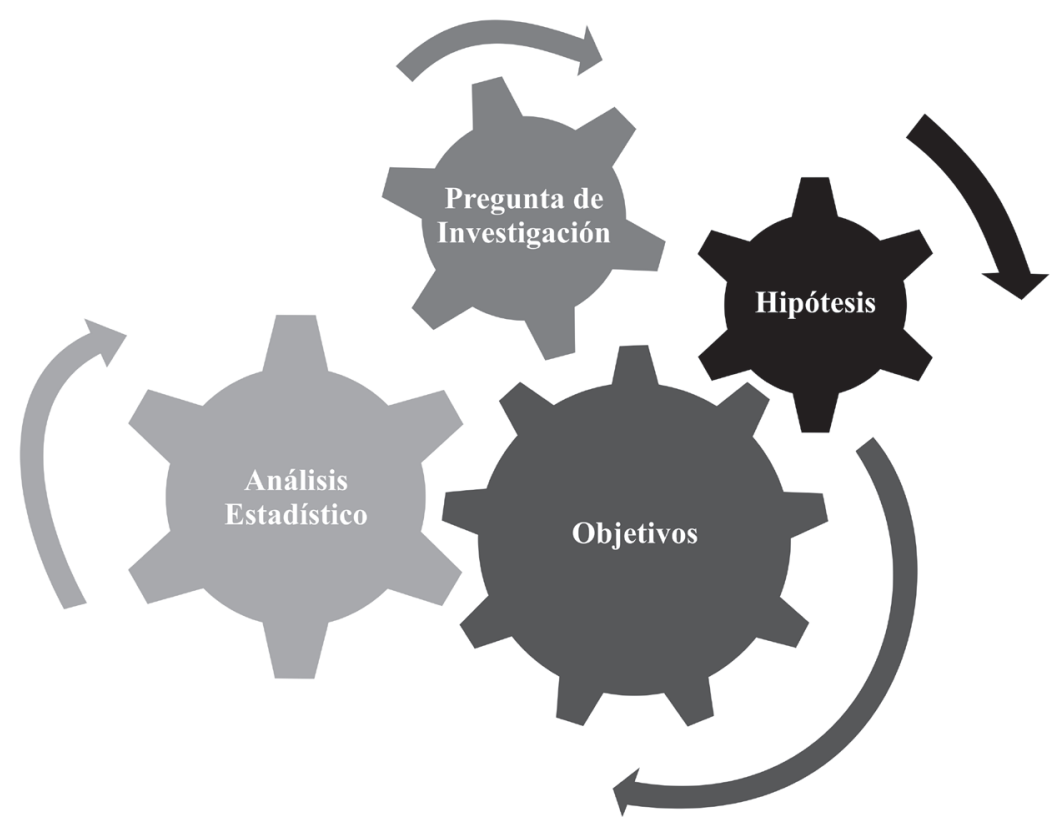

Figura 3. Relación entre la pregunta, hipótesis, objetivos y análisis estadístico de la investigación

Finalmente, es importante tener presente que este artículo se ha propuesto como una herramienta pedagógica con la cual se pueda comprender la relación existente entre los elementos básicos de una investigación, los cuales deben estar presentes de manera latente para que, como decía uno de mis grandes maestros, el trabajo de investigación sea completo, interrelacionado y pueda articularse de manera armoniosa (Díaz, 2014).

\section{REFERENCIAS}

Acevedo, I. (2002). Aspectos éticos en la investigación científica. Ciencia y Enfermería, 8(1), 15-18.

Arguedas, O. (2009). El ángulo del investigador. La pregunta de investigación. Acta Médica Costarricense, 51(2), 89-90.

Bologna, E. (2013). Estadística para Psicología y Educación, 3ra edición. Córdoba, Argentina: Brujas.

Carlson, N. (2014). Fisiología de la conducta, 11a edición. Madrid, España: Pearson Educación SA.

Castañeda, M., Cabrera, A., Navarro, Y., \& Vries, W. (2010). Procesamiento de datos y análisis estadísticos utilizando SPSS. Porto Alegre, Brasil: EDIPUCRS.
Creswell, J. (2014). Research Design. Qualitetive, Quantitative, and Mixed Methods Approaches, 4 Edition. London, United Kingdom: SAGE Publications Ltd.

Definición-ABC. (2018). Definición de Determinar. Recuperado el 23 de enero de 2018 de: https:// www.definicionabc.com/general/determinar.php.

Díaz, A. (2014). Comunicación personal sobre metodología de la investigación en psicología, noviembre de 2014. Concepción, Chile.

Doval,H. (2006). Acerca de la "imaginación científica" ¿Existe algún método para inventar ideas novedosas? Revista Argentina de Cardiología, 74(6), 505-511.

Field, A. (2009). Discovering Statistics Using SPSS. Third Edition. Los Angeles: SAGE.

Garrocho, J., \& Pozos, A. (2012). ¿De dónde surgen las preguntas de investigación? En A. Gordillo, U. Medina, \& M. Pierdant, Manual de Investigación Clínica (págs. 1-26). México D. F.: Manual Moderno.

Hernández, R., Fernández, C., \& Baptista, P. (2014). Metodología de la Investigación. Sexta Edición. México D.F.: McGraw-Hill / Interamericana Editores, S.A. de C.V. 
Hurtado, J. (2008). Cómo formular objetivos de investigación. Caracas, Venezuela: Quirón Ediciones.

Manterola, C., \& Otzen, T. (2013). Por qué investigar y cómo conducir una investigación. International Journal of Morphology, 31(4), 1498-1504.

Ramos-Galarza, C. (2015). Los paradigmas de la investigación científica. Avances en Psicología, $23(1), 9-17$.

Ramos-Galarza, C. (2016). La pregunta de investigación. Avances en Psicología, 24(1), 2331.

Ramos-Galarza, C. (2017 a). El abandono de la Estadística en la Psicología de Ecuador. Revista Chilena de Neuro-Psiquiatría, 55(2), 135-137. doi.org/10.4067/S0717-92272017000200008 .

Ramos-Galarza, C. (2017). Introducción a la investigación: entre anécdotas musicales y científicas. Quito, Ecuador: Don Bosco.
Real Academia de la Lengua Española. (2018). Concepto de la palabra espuria. Recuperado el 21 de enero de 2018 de: http://dle.rae. es/?id=GgGhUlr.

Real Academia de la Lengua Española. (2018). Definición de Determinar. Recuperado el 23 de enero de 2018 de: http://dle.rae.es/srv/ search? $\mathrm{m}=30 \& \mathrm{w}=$ determinar.

The Free Dictionary. (2018). Definición de determinar. Recuperado el 23 de enero de 2018 de: https:// es.thefreedictionary.com/determinar.

Word Reference. (2018). Definición de determinar. Recuperado el 23 de enero de 2018 de: http:// www.wordreference.com/definicion/determinar.

Fecha de recepción: 13 de marzo 2019

Fecha de aceptación: 22 de mayo 2019 